\title{
The effect of transcutaneous electrical acupoint stimulation on sleep quality in chronic insomnia disorder
}

\author{
Behnam Khaledipaveh $^{1,2}$, Habibolah Khazaie ${ }^{3}$, Nader Salari ${ }^{4}$, Soroush Maazinezhad ${ }^{5}$ \\ 1. Sleep Disorders Research Center, Kermanshah University of Medical Sciences, Kermanshah, Iran \\ 2. Department of psychiatric nursing, School of Nursing and Midwifery, Kermanshah University of Medical \\ Sciences, Kermanshah, Iran \\ 3. Sleep Disorders Research Center, Kermanshah University of Medical Sciences, Kermanshah, Iran \\ 4. biostatistics department, School of Health, Kermanshah University of Medical Sciences, Kermanshah, Iran \\ 5. Sleep Disorders Research Center, Kermanshah University of Medical Sciences, Kermanshah, Iran \\ (Corresponding author), Tel:083-37204071, Email: soroush.maazi@kums.ac.ir ,ORCID CD:0000-0001-8025- \\ 5334
}

\begin{abstract}
Background and Aim: Acupuncture is one of the most common methods of complementary medicine. The purpose of this study was to evaluate the effect of transcutaneous electrical acupoint stimulation on sleep quality in the patients with chronic insomnia.

Materials and Methods: This study was a single-blind clinical trial in which 40 patients with chronic insomnia were randomly placed into intervention (TEAS) and control (sham TEAS) groups. The acupuncture points including SP6, HT7 and LI4 were selected according to the medical guideline of traditional Chinese medicine. The stimulation was performed for 8 sessions, twice a week for one month. The Pittsburgh Sleep Quality Index (PSQI) and actigraphy were used to evaluate the patients' sleep quality before and after intervention.

Results: Results showed no significant differences in the mean scores of PSQI between the two groups before and after the intervention $(\mathrm{p}>0.05)$. In regard to the objective characteristics of sleep quality, total sleep time in the intervention group significantly increased $(p=0.015)$. These alterations were not significant in the sham TEAS group $(\mathrm{p}=0.502)$.

Conclusions: The results showed that TEAS can be effective in increasing the total sleep time in the patients with chronic insomnia disorder and this effect can be detectable by a monitoring instrument such as actigraphy.

Keywords: Actigraphy, Chronic insomnia, Pittsburgh Sleep Quality Index (PSQI), RCT, Sleep quality, Transcutaneous electrical acupoint stimulation
\end{abstract}

Received: feb16,2019 Accepted: Sep16.2019

How to cite the article: Behnam Khaledipaveh, Habibolah Khazaie, Nader Salari, Soroush
Maazinezhad. The effect of transcutaneous electrical acupoint stimulation on sleep quality in
chronic insomnia disorder. SJKU $2019 ; 24(5): 83-94$

Copyright (C) 2018 the Author (s). Published by Kurdistan University of Medical Sciences. This is an open access article distributed under the terms of the Creative Commons Attribution-Non Commercial License 4.0 (CCBY$\mathrm{NC}$ ), where it is permissible to download, share, remix, transform, and buildup the work provided it is properly cited. The work cannot be used commercially without permission from the journal. 


\title{
تأثير تحريك الكتريكى يوستى نقاط طب سوزنى بر كيفيت خواب در اختلال بىخوابى مزمن
}

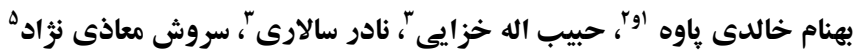

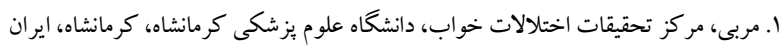

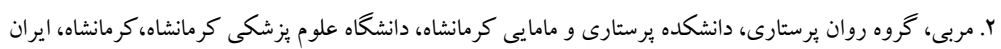

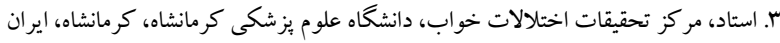

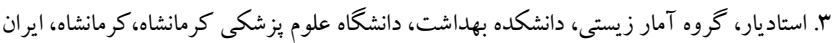

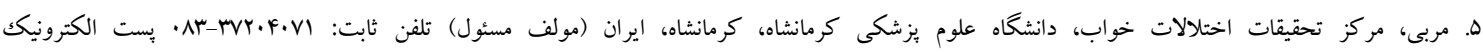

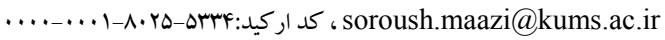

جكيده زمينه و هدف: طب سوزنى يكى از روش هاى رايج در حـوزه طب مكمـل اسـت. ايـن مطالعه بـا هـدف بررسى تـأثير تحريكك

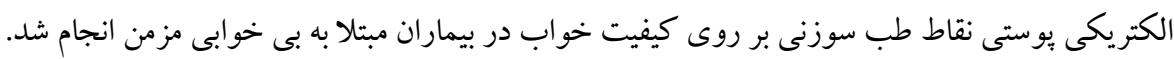

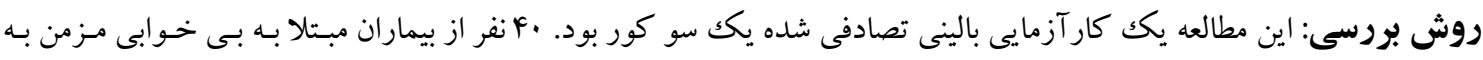

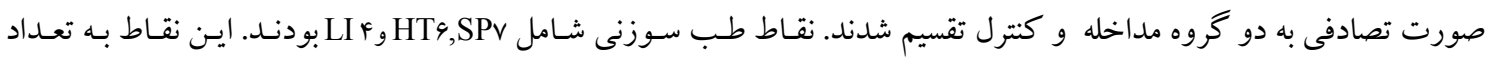

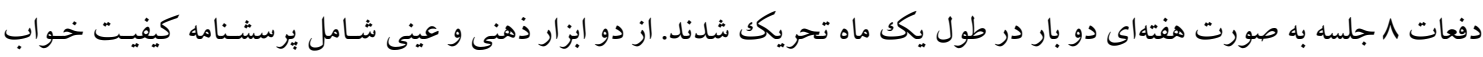

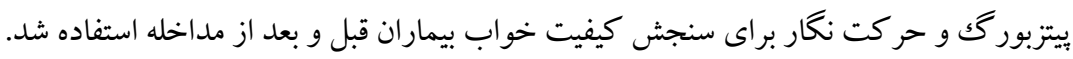

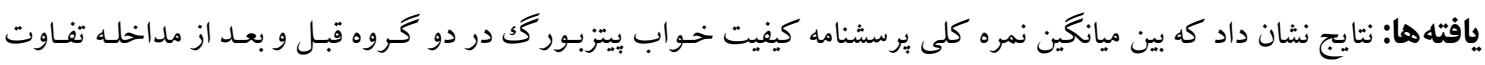

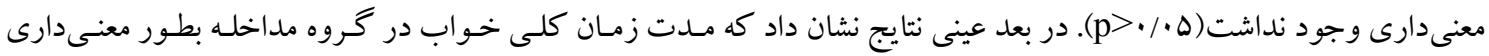

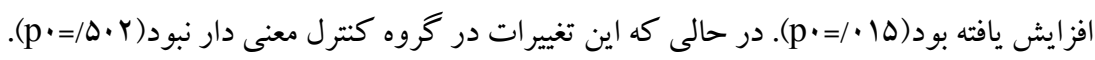

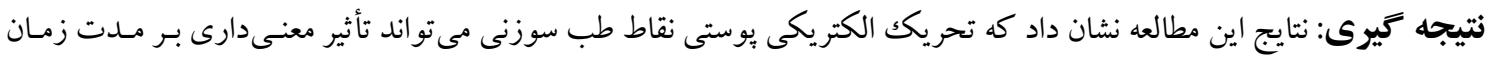



كلمات كليدى: بى خوابى مزمن، برسشنامه كيفيت خواب بيتزبورك،، كارَّمايى بالينى ، كيفيـت خواب، تحريـك الكتريكى يوستى نقاط طب سوزنى وصول مقاله:QV/II/TV 
اند(YF, YF). يكى از روش هاى غير دارويى كه مورد

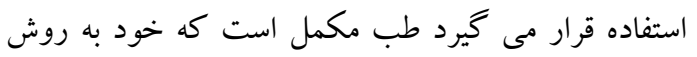
هاى درمانى يو كا، ماساز درمانى، گياه درمانى، هيينوتيزم، طب فشارى و طب سوزنى تقسيم بندى مى شود(Y)). در سال هاى اخير استفاده از روشهاى طب مكمل در درمان

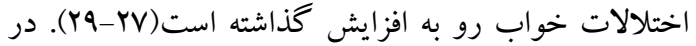
ميان اين روشها،طب سوزنى يكى از درمان هايى است كه

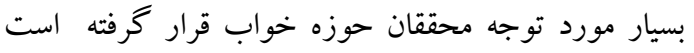

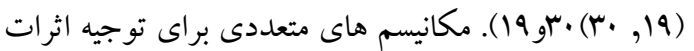
طب سوزنى بر وضعيت خواب مطرح شده اند كه مى توان

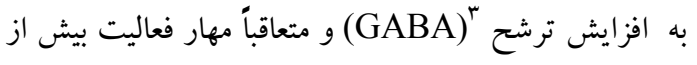
حد سيستم عصبى مر كزى بعد از درمان با طب سوزنى اشاره

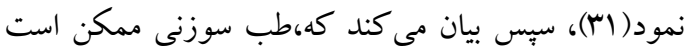

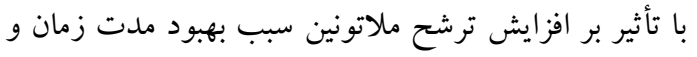

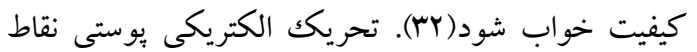

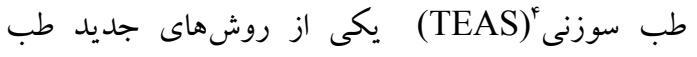

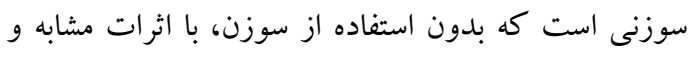

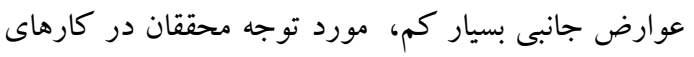

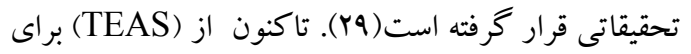
درمان مشكلاتى نظير خستخى تهوع استفراغ و درد استفاده

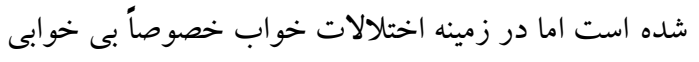

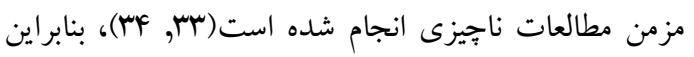

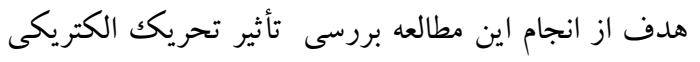
يوستى نقاط طب سوزنى بر كيفيت خواب در بيماران مبتلا به به خوابى مزمن است.

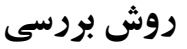
اين مطالعه يكك كار آزمايى بالينى تصادفى شده يكك سويه

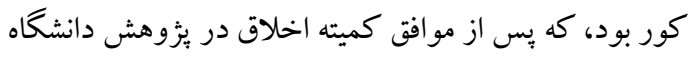

\footnotetext{
${ }^{3}$ Gamma-Aminobutyric acid

${ }^{4}$ Transcutaneous Electrical Acupoint Stimulation
}

بىخوابى يكك مشكل شايع در حوزه سلامت است(1). در بسيارى از كشورها برآورد شده است كه 9 تا • ا درصد مردم مبتلا به بى خوابى هستند(Y-Y). اين اختلال سالانه ميليون ها دلار ضرر بر اقتصاد كشورها وارد مى كند(ه). تعريف بىخوابى بر اساس دستهبندى بينالملى اختلالات

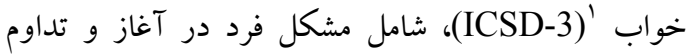
خواب، شرايط نامناسب براى به خواب رفتن و وجود رداب ييامدهاى روزانه بىخو ابى به مدت ب ماه و حداقل ب بار در

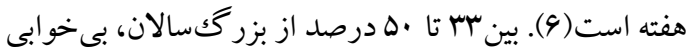
را به صورت كذرا بين جند روز تا جند هفته تجربه مى كنند

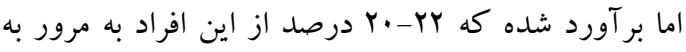

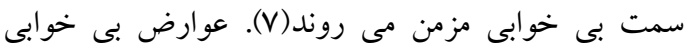

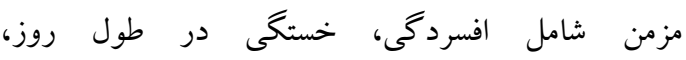
تحريكگيذيرى، اختلال عملكرد روزانه، مشكلات قلبى

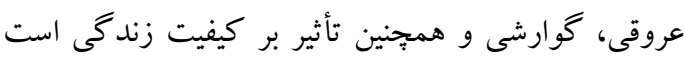

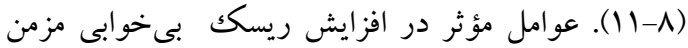

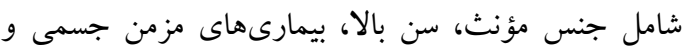

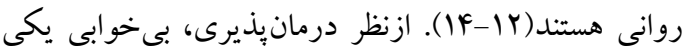

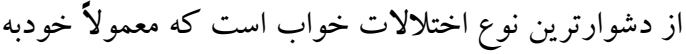
خود بهبود نمى يابد و درصد ناجيزى از مبتلايان به بـ خوابى مزمن درمان را بيخيخى مى كنند و به طور كامل درمان مىشوند(1), 19)19 19). تشخيص قطعى بىخوابى نيازمند اخذ يكك تاريخجه كامل و بررسى دقيق است(IV)، به منظور بررسى هاى ذهنى و عينى استفاده از يرسشنامه و دستخاه

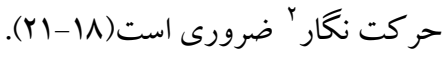
در طول سه دهه گذشته درمان دارويى بى خوابى مورد

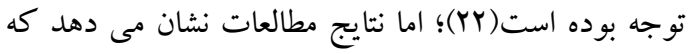

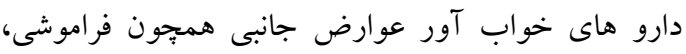

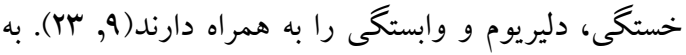

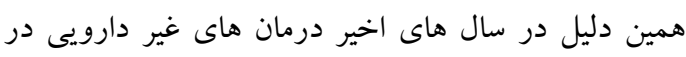

\footnotetext{
${ }^{1}$ International Classification of Sleep Disorders

${ }^{2}$ Actigraphy
} 
خواب، كيفيت خواب آنان توسط برسشنامه كيفيت خواب ييتزبور گك و حر كت نكارى ثبت شـد. تحريكك الكتريكى يوسـى نقـــاط طـب سـوزنى بـه وسـيله دستـاه(TEAS) برند)(ACUHEALTH) مدل (proq..) با شماره سريال) ساخت استراليا انجام شد. اين دستخاه بـا قابليت c=rrVD نقطه يابى (AcuPoint Findeing) قادر به ويدا كردن نقاط

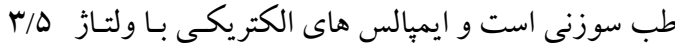

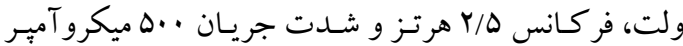
وارد مى نمايد. بعد از تماس دادن يـروب دستخاه بـا يوست فرتح

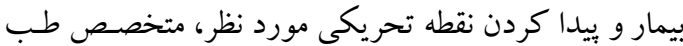

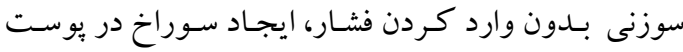

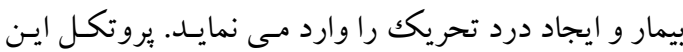
مطالعه از مقاله قنبرى زاده و همكاران الكَو بـردارى شـد. در

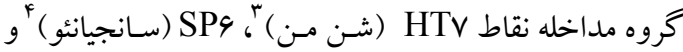
LIF شكل ا، نشاندهنده موقعيت آناتوميكى نقاط هستـند. ايـن كار براى هر فرد به تعداد دفعات ^ جلسه و هر بار به مـدت هـ دقيقه (هر نقطه •ه ثانيه)، توسط متخصص طـب سـوزنى در مركز تحقيقات اختلالات خواب انجام شد. در گروه كنترل،

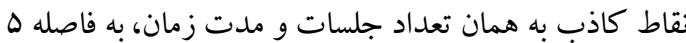
الى •ا سـانتى متر از نقـاط واقعى توسط دستخاه تحريكك شدند. ابزار مورد استفاده براى سنجش كيفيت خواب ذهنى الفي بيماران يرسشنامه كيفيت خواب ييتزبور گ(PSQI) بود. اين

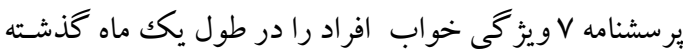
مشخص مى سازد. ايـن مـوارد عبـارتانـد از: كيفيـت ذهنى

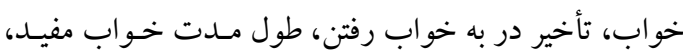
كفايست خــواب، اخستلالات خـواب، اسـتفاده از داروهـاى

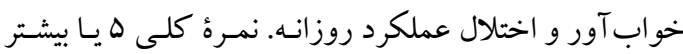
نشاندهندة كيفيت خواب نامطلوب اسـت. ايـن يرسشـنامه از

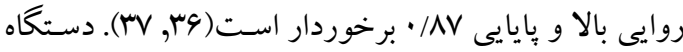

\footnotetext{
${ }^{2}$ Actigraphy

${ }^{3}$ Shen Men

${ }^{4}$ Sanyingjiao

${ }^{5}$ Hugo
}

علوم يزشكى كرمانشاه و ثبت در سامانه ملى كارآزمايى

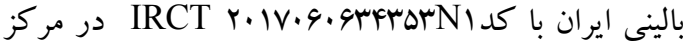
تحقيقات اختلالات خواب دانشگاه علوم يزشكى كرمانشاه

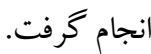
شر كت كنند گان يس از اخذ رضايت نامه آكًانه وارد مطالعه شدند. معيار هاى ورود شامل: داشتن تشخيص بىخوابى مزمن بر طبق معيار هاى( س-ICSD) ، تشخيص بزشك متخصص اعصاب و روان، داشتن امتياز ه و بالاتر در يرسشنامه كيفيت خواب بيتزبورگك، تمايل جهت شركت در مطالعه، عدم جراحت و قطع شدن اندامهاى فوقانى و تحتانى، نداشتن ضربان ساز، عدم وارد شدن استرس روانى شديد در و ماه كذشته(آسيب و جراحت شديد، فوت عزيزان)، عدم اعتياد به مواد مخدر و عدم ابتلا به بيمارىهاى بدخيم بود. باردارى در زمان مطالعه، ابتلا به بيمارى جسمى و روانى حاد در زمان شركت در مطالعه و عدم تمايل به ادامه همكارى معيار هاى خروج از اين مطالعه بود. حجم نمونه بر اساس فرمول مقايسه يكك صفت كمى در ب گروه و بر مبناى يارامترهاى آن يعنى سطح اطمينان ه9 ٪)، توان

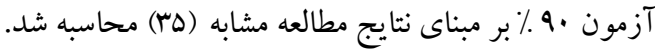

با توجه به امكان مواجهه با ريزش در بـين شـركت كنــدكان در طى مراحل اين مطالعهه و همجنـين افز ايش تـوان آزمـون

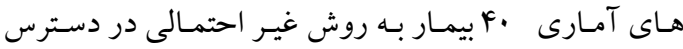
انتخاب و به صورت تصادفى بـه دو گـروه مداخله و كنتـرل تقسـيم بنــدى شــدند. بــا اسـتفاده از روش ينهــان ســازى

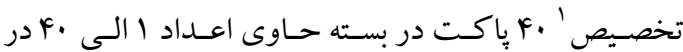
اختيار بيمـاران قـرار گرفـت. اعـداد زوج (كروه مداخلـه) و اعداد فرد (كروه كنترل) و بر اين اساس بيماران به دو گحروه مداخله (TEAS) و كنترل (SHAM TEAS) يـا تحريـك

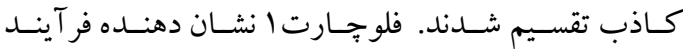
تخصـيص شـر كت كنــد گان در مطالعـه اسـت. در ابتـداى مراجعـه، شـر كت كنتـد كان بـه مر كـز تحقيقـات اخـتلالات

${ }^{1}$ Allocation Concealment 
جدول ا. محل آناتوميكى نقاط طب سوزنى

\begin{tabular}{|c|c|}
\hline محل آناتوميكى & نقاط طب سوزنى \\
\hline $\begin{array}{c}\text { ه سانتى متر بالاتر از قوز داخك } \\
\text { داخلى }\end{array}$ & Sp6 (Sanyingjiao) \\
\hline در دستها روى استخوان & HT7 ( shen men) \\
\hline در قسمت نرمه بين انگشت & LI4 (hugo) \\
\hline
\end{tabular}
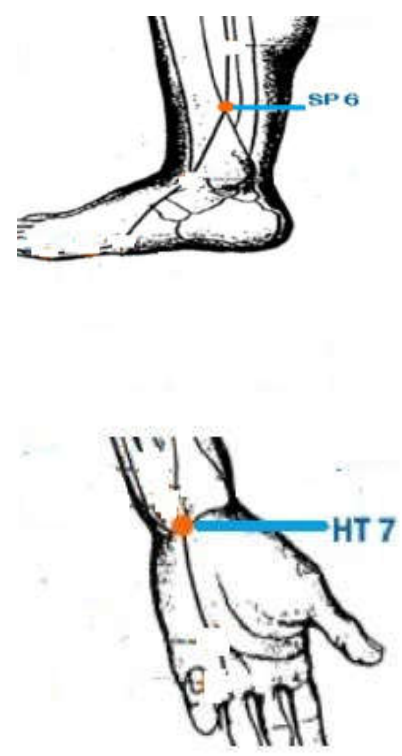

شكل ا. محل آناتوميكى نقاط طب سوزنى بر اساس تصوير
حر كت نغار (اكتى گراف) ساخت شركت (Monitoring) (Ambulatory بيماران بـه صـورت(Real Time) قبـل و بعـد از مداخله استفاده شد. اين دستكاه كه به صورت قابل حمل و در ابعاد يكت ساعت مجِى است به فرد بيمار داده مىشود. دستخاه بـا ثبت حر كات بيمار به صورت شبانه روز مـدت زمـان خـواب شبانه را ثبت مى نمايد. طهماسيان و همكاران (•r)در سـال 19در يكك مقاله مرورى بيان كردند كه حركت نغخارى بـه عنوان يكك روش دقيق و عينى در ثبـت مـدت زمـان خـواب شبانه بيماران مورد اعتماد است واحد هـاى يـرُوهش، انــازه كيرى و ثبت گرديد. به شر كت كنند كان آموزش داده شـد

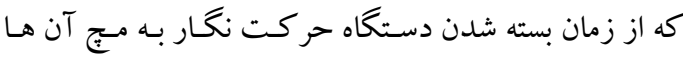
توسط تيم تحقيق، بـه مـدت هـ شبانه روز ييوسته آن را را بـاز

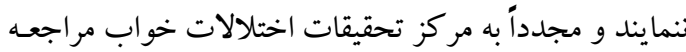

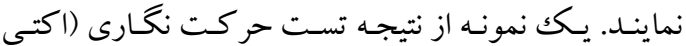

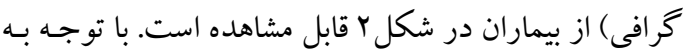

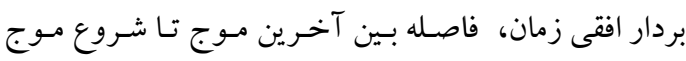

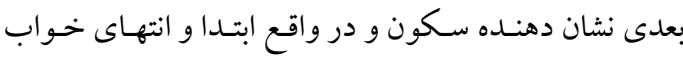
بيمار است كه توسط نرم افزار ه. Action watch V.Y بـهـ

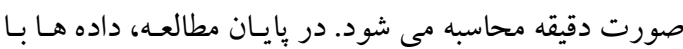

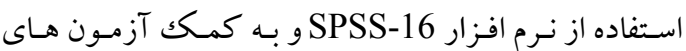

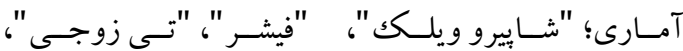

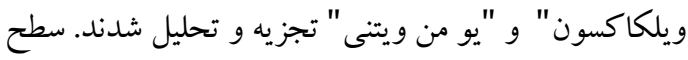

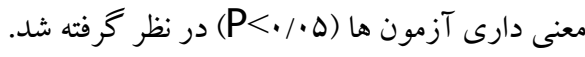



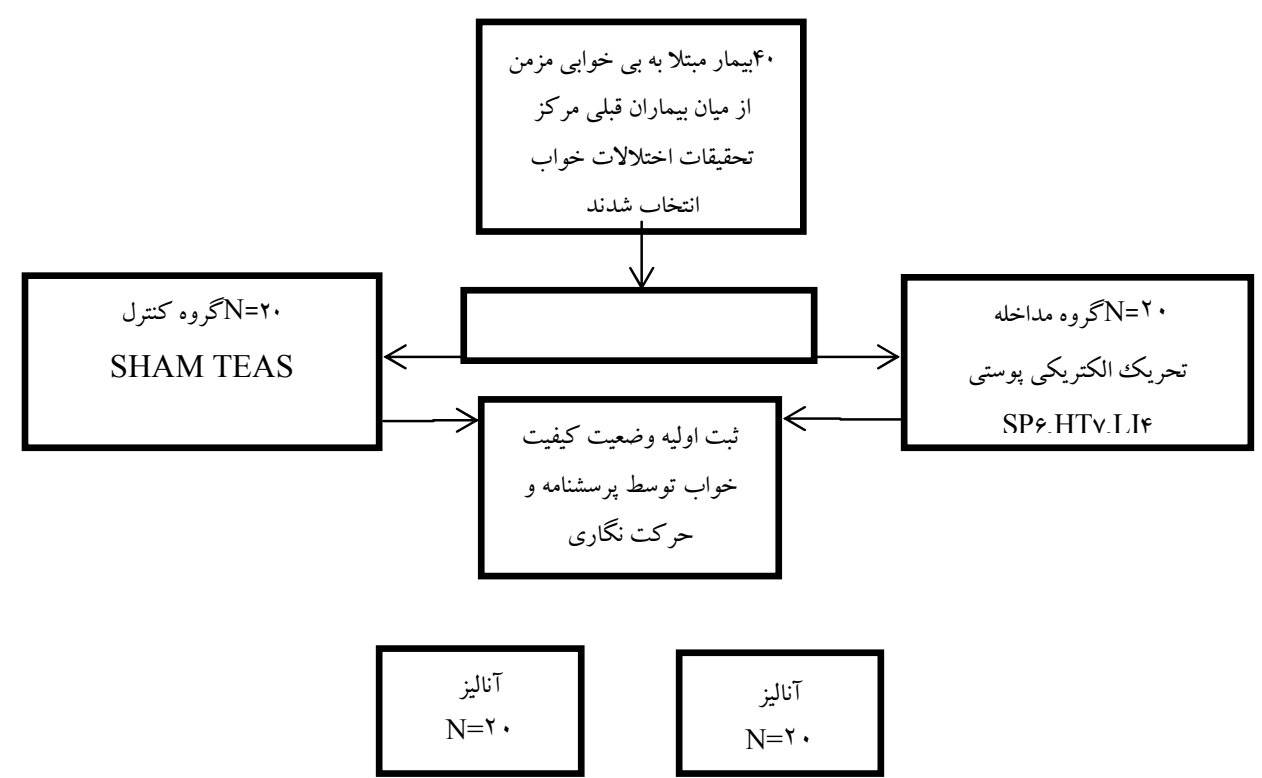

فلوجارت ا. فر آيند تخصيص شر كت كنند كان به دو گروه

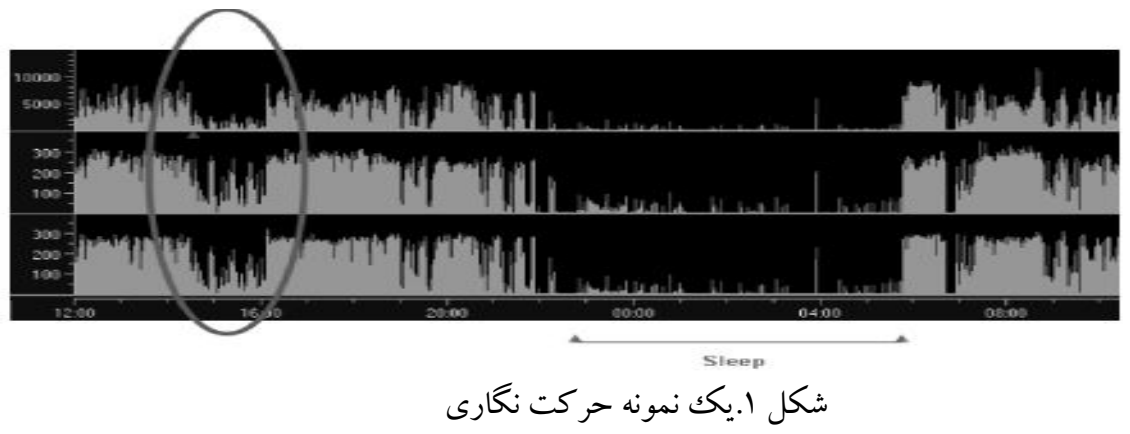

نتايج يرسشنامه كيفيت خواب بيتزبورك نشان داد كه بعـد

كافته ها

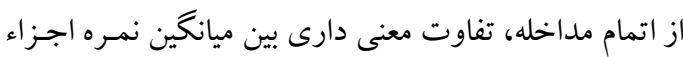
كليه شر كت كنند گان تا بايان مطالعه به همكارى خود ادامه

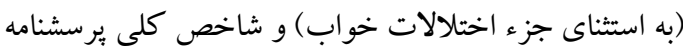

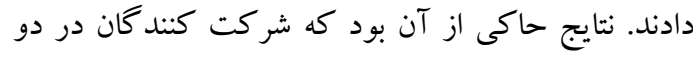
كيفيـت خـواب ييتزبـورگك در دو گـروه مداخلـه و كنتـرل گروه مداخله و كنترل از نظر مشخصات دمو گرافيك سن،

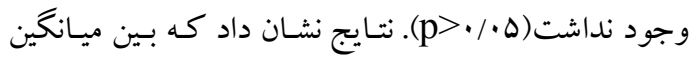

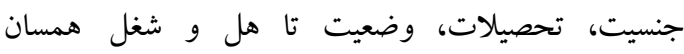

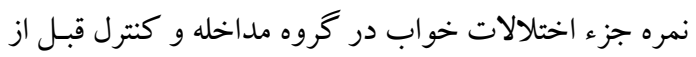
بودند(ه//p>). در گروه مداخله ها درصد و در گروه مداخله تفاوت معنادارى وجود نداشت( كنترل •F درصد از شركت كنند كان مرد بودند. ميانكين از مداخله بين دو گرووه تفـاوت آمـارى معنـادارى مشـاهده

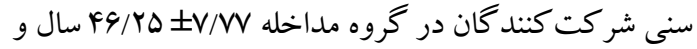

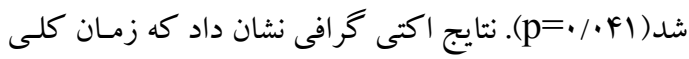
در گروه كنترل

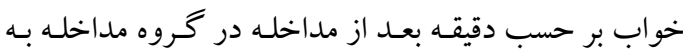

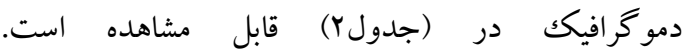


است كه مقدار تغييـرات ميـانگين (TST) بعـد از مداخلـه در

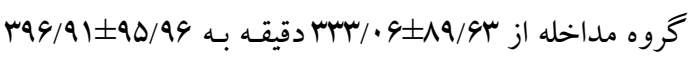
دقيقه تغيير يافت در حالى كه ايـن تغيير در گرووه كنترل از

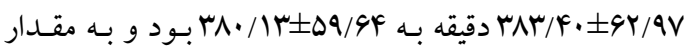
تقريبى ب دقيقه كاهش يافته بود ـ نتايج آزمـون هـاى آمـارى در (جدول r) قابل مشاهده است.

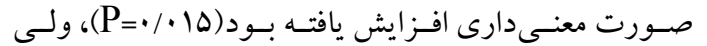
تغييرات ميانگين قبل و بعد از مداخله ايسن يـارامتر از مداخلـه

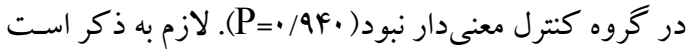
كه ميانگين متغيير زمان كلى خواب(TST) در گروه كنترل قبل از شروع مداخله به صسورت تصـادفى از ميـانگين مـدت زمان خواب در گروه مداخله بيشتر بود و اين يارامتر قبـل از مداخله در بين دو گروه همسان نبود. اما نكته قابل توجه ايـن

\begin{tabular}{|c|c|c|c|}
\hline & $v$ & r & دانشكاهى \\
\hline \multirow{3}{*}{$\cdot / \Delta . . * *$} & & & وضعيت تأهل \\
\hline & IV & 19 & متأهل \\
\hline & $r$ & r & مجرد \\
\hline \multirow[t]{3}{*}{$\cdot / W V^{* * *}$} & & & شغل \\
\hline & ir & ir & بيكار \\
\hline & $\wedge$ & v & كارمند \\
\hline
\end{tabular}

آزمون تى زوجى ***زمون فيشر 
جدول ب. مقايسه تغييرات ميانكين نمرات اجزاء برسشنامه كيفيت خواب بيتزبور گك و حركت نگارى قبل و بعد از مداخله در گروه مداخله و

كنترل

\begin{tabular}{|c|c|c|c|c|c|c|c|c|}
\hline \multirow[t]{2}{*}{$P^{c}$} & \multirow[t]{2}{*}{$P^{b}$} & \multirow[t]{2}{*}{$P^{a}$} & \multicolumn{2}{|c|}{ كروه كنترل } & \multirow[t]{2}{*}{$P^{a}$} & \multicolumn{2}{|c|}{ كروه مداخله } & \multirow{2}{*}{ كيتز } \\
\hline & & & انحراف معيار ثميانخين & انحراف ثميانخين & & انحراف ثميانكين & انحراف ثميانخين & \\
\hline . & . N91 & $\% \cdots 1$ & $1 / 9 \cdot \pm \cdot / N r$ & 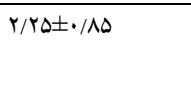 & $<\cdot / \cdot \cdot 1$ & $1 / r \cdot \pm \cdot / v r$ & $r / r \Delta \pm \cdot / v \Delta$ & خويفيت ذهنى \\
\hline$\cdot / 1 \cdot 1$ &.$/$ TqF &.$/ .14$ & $r \pm \cdot / 94$ & $r / \uparrow \Delta \pm \cdot / \Delta$. & $<\cdot / \cdot \cdot 1$ & $1 / 9 \Delta \pm \cdot / 91$ & $r / 90 \pm \cdot / 4 q$ & نهفتخى خواب \\
\hline 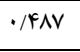 & .rar & $\% r$ & $1 / N \cdot \pm \cdot / A$ & $r / r \cdot \pm \cdot / r r$ & 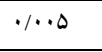 & $1 / 9 \cdot \pm \cdot / v \wedge$ & $r / \Delta \cdot \pm \cdot / 99$ & طول خواب \\
\hline$\cdot / \pi r V$ & $\cdot / \Delta F \Delta$ &.$/ .14$ & $1 / 90 \pm 1 / \cdot 1$ & $r / \Delta q \pm \cdot / \wedge q$ & $\cdot / \cdot r$ & $Y / \cdot \Delta \pm 1 / \cdot q$ & $r / 9 \cdot \pm \cdot / M$ & اثر بخشى خواب \\
\hline $.1 .+1$ & $\cdot / 190$ & $\cdot / T F A$ & $1 / F \Delta \pm \cdot / \Delta 1$ & $1 / 90 \pm \cdot / 09$ & $\cdot / \cdots \wedge$ & $1 / 1 \Delta \pm \cdot / r V$ & $1 / 90 \pm \cdot / 9 \mathrm{~V}$ & اختلالات خواب \\
\hline$\cdot / r \cdot V$ &.$/ 49 V$ & $\cdot / V / 9$ & $1 / A \cdot \pm 1 / \cdot 1$ & $|/ 9 \cdot \pm| / Y 1$ & $\cdot / v \cdot \Delta$ & $r / \backslash \Delta \pm ו / \backslash \Lambda$ & $r / 1 \cdot \pm 1 / r q$ & خواب آور از دارو \\
\hline$\cdot / r V$ &.$/ 990$ & .1 .4 & $1 / Y \Delta \pm \cdot / \mathrm{VV}$ & $1 / v \cdot \pm \cdot / 91$ &.$/ .9$ & $1 / r \cdot \pm \cdot / \Delta V$ & $1 / V \cdot \pm \cdot / \wedge 9$ & روزانه \\
\hline$\cdot / A V$ &.$/ 19 V$ & $\% r$ & $11 / f \cdot \pm r / 10$ & $\mid F / \Lambda \cdot \pm r / \cdot r$ & $<\cdot / \cdot \cdot 1$ & $\| / V \cdot \pm r / F \mid$ & $1 Q / \Lambda \cdot \pm r / V \Lambda$ & $\begin{array}{r}\text { امتياز كلى برسشنامه } \\
\text { PSQI }\end{array}$ \\
\hline - &.$/ .4 F$ &.$/ 94$. & $r \Lambda \cdot / I r \pm \Delta q / q r$ & $r \Delta r / 4 \cdot \pm G r / q V$ & $\because \% \cdot 10$ & $r 99 / 9 \cdot \pm q \Delta / 9 \varphi$ & $\Gamma r / q \mu \pm \wedge q / q \mu$ & 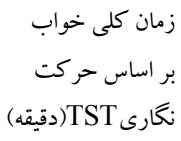 \\
\hline
\end{tabular}

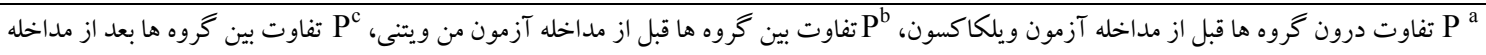

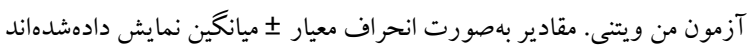


سوزن بود. براى سنجش كيفيت خواب بيماران از حركت

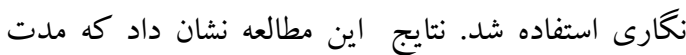
زمان كلى خواب و ترشح هورمون ملاتونين در گروه

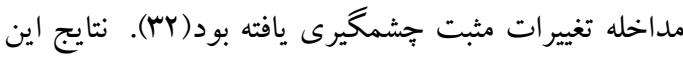

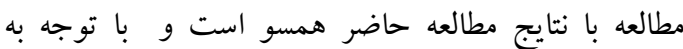
مشاهده اثر كذارى طب سوزنى بر ترشح هورمون ملاتونين كه از عوامل مهم در بهبود كيفيت خواب است مىتواند اثرات فيزيولوزيك طب سوزنى بر سيسم عصبى مركزى را

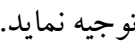

Chung و همكاران در سال 19.r يك كار آزمايى بالينى يكك سويه كور با هدف بررسى تأثير طب سوزنى الكتريكى

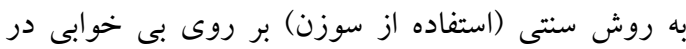
بيماران مبتلا به افسردكى اساسى، به نتايجى دست بيدا كردند كه غيرهمسو با مطالعه حاضر بود. آنها بعد از اتمام مداخله نتايج معنىدارى در كيفيت خواب بيماران مشاهده نكردند. اين مطالعه از نظر روش كار و نقاط تحريكك با لنائ مطالعه حاضر متفاوت بود. به نظر مى رسد وجود اختلال افسردگى اساسى(MDD) در شر كت كنند كان اين مطالعه به

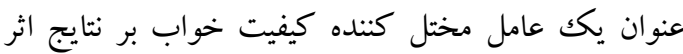
كذار بوده است( (rq).

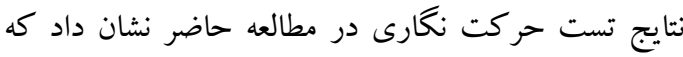
به صورت معنى دار مدت زمان خواب شبانه بيماران را در گروه مداخله افزايش داده بود، اما در گروه كنترل تغييرات معنى دار مشاهده نشد. با اين حال نتايج سنجش ذهنى كيفيت خواب در هر دو كروه از بيماران

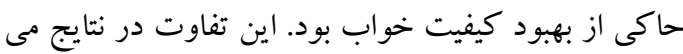

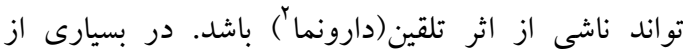
مطالعات كار آزمايى امكان دارد كه تغييرات ايجاد شده در العرد فرد بيمار به دنبال انجام يكك روش درمانى، ناشى از اثر تلقين باشد. Dupont و همكاران يكك مطالعه تجربى در سال

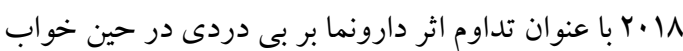

\footnotetext{
${ }^{2}$ Placebo Effect
}

يافته هاى يُزوهش در بعد ذهنى (يرسشنامه) حاكى از آن بود

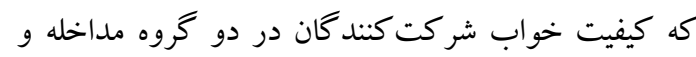
كنترل بهصورت معنى دار افزايش يافته بود، ولى با استفاده

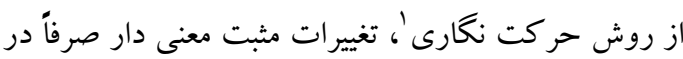

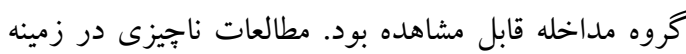

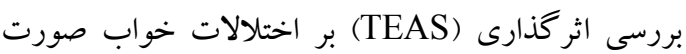
كرفته است. قنبرى زاده و همكاران در سالVه يك كارآزمايى بالينى يك سويه كور با هدف بررسى اثر (TEAS) بر كيفيت

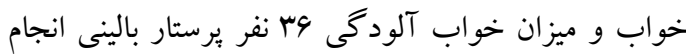

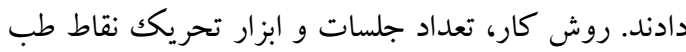
سوزنى با مطالعه حاضر مشابه بود، اما براى سنجش كيفيت

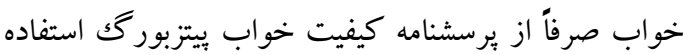
شده بود. نتايج اين مطالعه نشان داد كه (TEAS) در گروه بره مداخله تأثير معنىدارى بر ابعاد ذهنى كيفيت خواب ابن و كاهش خوابآلودگى روزانه برستاران گذاشته بود(ها).

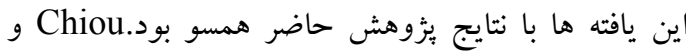

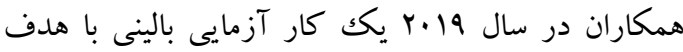
بررسى اثر گذارى (TEAS) بر شدت دردهاى عصبى، خلق و كيفيت خواب بر روى ع 4 بيمار با ضايعه نخاعى انجام

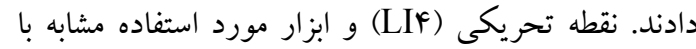
مطالعه حاضر بود. نتايج اين مطالعه نشان داد كه (TEAS)

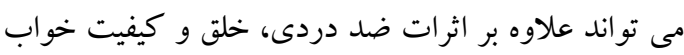

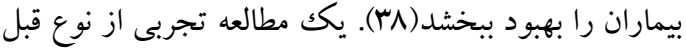
و بعد توسط Spence و همكاران در سال F...F با هدف بررسى اثرات طب سوزنى الكتريكى بر كيفيت خواب، اضطراب و ميزان ترشح هورمون ملاتونين در ل1 ل بيمار بزرگسال انجام شد. تعداد جلسات تحريك نقاط طب نهر سوزنى هفتهاى دو بار به مدت هـ هفته بود و ابزار مورد



\footnotetext{
${ }^{1}$ Actigraphy
} 


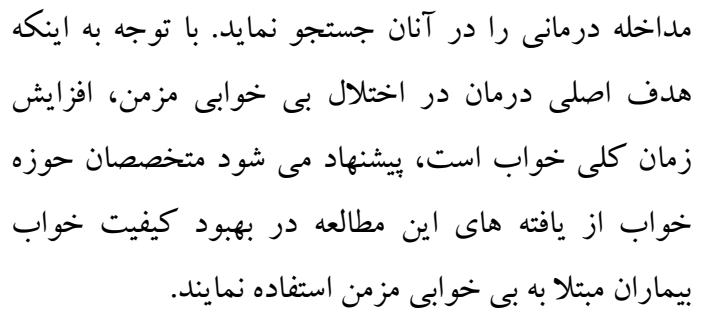

\section{Refrences}

1.Torrens I, Argüelles-Vázquez R, Lorente-Montalvo P, Molero-Alfonso C, Esteva M. Prevalence of insomnia and characteristic of patients with insomnia in a health area of Majorca (Spain). Atencion primaria. 2019.

2.Klingaman EA, Brownlow JA, Boland EM, Mosti C, Gehrman PR. Prevalence, predictors and correlates of insomnia in US army soldiers. Journal of sleep research. 2018;27(3):e12612. 3.Nowicki Z, Grabowski K, Cubała WJ, Nowicka-Sauer K, Zdrojewski T, Rutkowski M, et al. Prevalence of self-reported insomnia in general population of Poland. Psychiatr Pol. 2016;50(1):165-73.

4.Kim KW, Kang S-H, Yoon I-Y, Lee SD, Ju G, Han JW, et al. Prevalence and clinical characteristics of insomnia and its subtypes in the Korean elderly. Archives of gerontology and geriatrics. 2017;68:68-75.

5.Kessler RC, Berglund PA, Coulouvrat C, Hajak G, Roth T, Shahly V, et al. Insomnia and the performance of US workers: results from the America insomnia survey. Sleep. 2011;34(9):1161-71.

6.Sateia MJ. International classification of sleep disorders. Chest. 2014;146(5):1387-94.

7.Yan J, Jiaming Z. Research on the Relationship between Insomnia and Illness Based on Multiple Logistic Regression Methods. Natural Science Journal of Harbin Normal University. 2018(1):3.

8.Lin Y-f, Liu Z-d, Ma W, Shen W-d. Hazards of insomnia and the effects of acupuncture treatment on insomnia. Journal of integrative medicine. 2016;14(3):174-86. 
9.Feyzabadi Z, Jafari F, Kamali SH, Ashayeri H, Aval SB, Esfahani MM, et al. Efficacy of Viola odorata in treatment of chronic insomnia. Iranian Red Crescent Medical Journal. 2014;16(12.(

10.Ishak WW, Bagot K, Thomas S, Magakian N, Bedwani D, Larson D, et al. Quality of life in patients suffering from insomnia. Innovations in clinical neuroscience. 2012;9 1 r.:( $(\cdot)$

11.Rezaie L, Khazaie H, Yazdani F. Exploration of the experience of living with chronic insomnia: A qualitative study. Sleep Science. 2016;9(3):179-85.

12.Babson KA, Wong AC, Morabito D, Kimerling R. Insomnia symptoms among female veterans: prevalence, risk factors, and the impact on psychosocial functioning and health care utilization. Journal of Clinical Sleep Medicine. 2018;14(06):931-9.

13.Uhlig BL, Sand T, Nilsen T, Mork PJ, Hagen K. Insomnia and risk of chronic musculoskeletal complaints :longitudinal data from the HUNT study, Norway. BMC musculoskeletal disorders. 2018;19(1):128.

14.Nishitani N, Kawasaki Y, Sakakibara H. Insomnia and depression: risk factors for development of depression in male Japanese workers during 2011-2013. International journal of public health. 2018;63(1):49-55.

15.Khazaie H, Rezaei L, Tahmasian M, Ahmadi A, Schwebel D, Russo M. insomnia treatment by olanzapin is sleep state misperception a psychotic disorder?(a case report). sleep Medicine. 2009;10(2):18-2.

16.Pigeon WR. Diagnosis, prevalence, pathways, consequences \& treatment of insomnia. The Indian journal of medical research. 2010;131:321-32.

17.Albrecht JS, Wickwire EM, Vadlamani A, Scharf SM, Tom SE. Trends in Insomnia Diagnosis and Treatment Among Medicare Beneficiaries, 2006-2013. The American Journal of Geriatric Psychiatry. 2019;27(3):301-9.

18.Pinto Jr LR, Bittencourt LRA, Treptow EC, Braga LR, Tufik S. Eszopiclone versus zopiclone in the treatment of insomnia. Clinics. 2016;71(1):5-9.

19.Mandiroglu S, Ozdilekcan C. Impact of Acupuncture on Chronic Insomnia: A Report of Two Cases with Polysomnographic Evaluation. Journal of acupuncture and meridian studies. 2017;10(2):135-8.

20.Tahmasian M, Khazaie H, Sepehry AA, Russo MB. Ambulatory monitoring of sleep disorders. J Pak Med Assoc. 2010;60(6):480-7.

21.Tahmasian M, Khazaie H, Golshani S, Avis KT. Clinical application of actigraphy in psychotic disorders: a systematic review. Current psychiatry reports. 2013;15(6):359.

22.Frase L, Nissen C, Riemann D, Spiegelhalder K. Making sleep easier: pharmacological interventions for insomnia. Expert opinion on pharmacotherapy. 2018;19(13):1465-73.

23.Andalib S, Vaseghi A, Vaseghi G, Naeini AM. Sedative and hypnotic effects of Iranian traditional medicinal herbs used for treatment of insomnia. EXCLI journal. 2011;10:192-7.

24.Guthrie KA, Larson JC, Ensrud KE, Anderson GL, Carpenter JS, Freeman EW, et al. Effects of pharmacologic and nonpharmacologic interventions on insomnia symptoms and self-reported sleep quality in women with hot flashes: a pooled analysis of individual participant data from four MSFLASH trials. Sleep. 2018;41(1):Zsx190.

25.Manber R, Bei B, Simpson N, Asarnow L, Rangel E, Sit A, et al. Cognitive Behavioral Therapy for Prenatal Insomnia: A Randomized Controlled Trial. Obstetrics and gynecology. 2019;133(5):911.

26.Micozzi MS. Fundamentals of Complementary, Alternative, and Integrative Medicine-EBook: Elsevier Health Sciences; 2018.

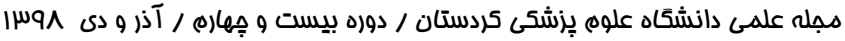


27.Chung K, Yeung W, Zhang S, Zhang Z, Wong M, Lee W, et al. Acupuncture for persistent insomnia associated with major depressive disorder: a randomised controlled trial. Hong Kong Medical Journal. 2016.

28. Yeung W-F, Chung K-F, Leung Y-K, Zhang S-P, Law AC. Traditional needle acupuncture treatment for insomnia: a systematic review of randomized controlled trials. Sleep medicine. 2009;10(7):694-704.

29.Yeung W-F, Chung K-F, Zhang S-P, Yap T-G, Law AC. Electroacupuncture for primary insomnia: a randomized controlled trial. Sleep. 2009;32(8):1039-47.

30.Ernst E, Lee MS, Choi T-Y. Acupuncture for insomnia? An overview of systematic reviews. The European journal of general practice. 2011;17(2):116-23.

31.Shergis JL, Ni X, Jackson ML, Zhang AL, Guo X, Li Y, et al. A systematic review of acupuncture for sleep quality in people with insomnia. Complementary therapies in medicine. 2016;26:11-20.

32. Spence DW, Kayumov L, Chen A, Lowe A, Jain U, Katzman MA, et al. Acupuncture increases nocturnal melatonin secretion and reduces insomnia and anxiety: a preliminary report. The Journal of neuropsychiatry and clinical neurosciences. 2004;16(1):19-28.

33. So RC, $\mathrm{Ng} \mathrm{JK}-\mathrm{F}, \mathrm{Ng}$ GY. Effect of transcutaneous electrical acupoint stimulation on

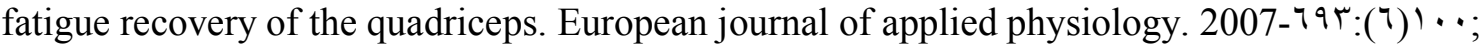
$\checkmark \cdots$.

34.Habib AS, Itchon-Ramos N, Phillips-Bute BG, Gan TJ. Transcutaneous acupoint electrical stimulation with the ReliefBand ${ }^{\circledR}$ for the prevention of nausea and vomiting during and after cesarean delivery under spinal anesthesia. Anesthesia \& Analgesia. 2006;102(2):581-4.

35.Qanbari Zadeh B, Hadadian F, Salari N, Maazinezhad S, Khaledi Paveh B. The Effect of Transcotaneus Electrical Acupoint Stimulation on Sleep Quality in Nurses. J Kermanshah Univ Med Sci. 2018; In Press(In Press):e68669.

36.Backhaus J, Junghanns K, Broocks A, Riemann D, Hohagen F. Test-retest reliability and validity of the Pittsburgh Sleep Quality Index in primary insomnia. Journal of psychosomatic research. 2002;53(3):737-40.

37.Buysse DJ, Reynolds CF, Monk TH, Berman SR, Kupfer DJ. The Pittsburgh Sleep Quality Index: a new instrument for psychiatric practice and research. Psychiatry research. 1989;28(2):193-213.

38.Chiou Y-F, Yeh M-L, Wang Y-J. Transcutaneous Electrical Nerve Stimulation on Acupuncture Points Improves Myofascial Pain, Moods, and Sleep Quality. Rehabilitation nursing: the official journal of the Association of Rehabilitation Nurses. 2019.

39.Chung K, Yeung W, Zhang S. Acupuncture for persistent insomnia associated with major depressive disorder. HEALTH AND HEALTH SERVICES RESEARCH FUND. 2016;22(1):9-14.

40.Laverdure-Dupont D, Rainville P, Renancio C, Montplaisir J, Lavigne G. Placebo analgesia persists during sleep: An experimental study. Progress in Neuro-

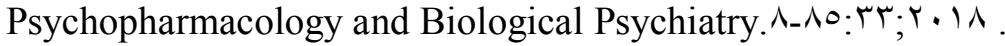

